University of Nebraska - Lincoln

DigitalCommons@University of Nebraska - Lincoln

\title{
Conceptual model of sediment processes in the upper Yuba River watershed, Sierra Nevada, CA
}

Jennifer A. Curtis

U.S. Geological Survey, jacurtis@usgs.gov

Lorraine E. Flint

U.S. Geological Survey

Charles N. Alpers

U.S. Geological Survey, cnalpers@usgs.gov

Sarah M. Yarnell

University of California - Davis

Follow this and additional works at: https://digitalcommons.unl.edu/usgsstaffpub

Part of the Earth Sciences Commons

Curtis, Jennifer A.; Flint, Lorraine E.; Alpers, Charles N.; and Yarnell, Sarah M., "Conceptual model of sediment processes in the upper Yuba River watershed, Sierra Nevada, CA" (2005). USGS Staff -Published Research. 482.

https://digitalcommons.unl.edu/usgsstaffpub/482

This Article is brought to you for free and open access by the US Geological Survey at DigitalCommons@University of Nebraska - Lincoln. It has been accepted for inclusion in USGS Staff -- Published Research by an authorized administrator of DigitalCommons@University of Nebraska - Lincoln. 
science@ $d$ irect

Geomorphology 68 (2005) 149-166
GEPMOPPHODGY

www.elsevier.com/locate/geomorph

\title{
Conceptual model of sediment processes in the upper Yuba River watershed, Sierra Nevada, CA
}

\author{
Jennifer A. Curtis ${ }^{\mathrm{a}, *}$, Lorraine E. Flint ${ }^{\mathrm{a}}$, Charles N. Alpers ${ }^{\mathrm{a}}$, Sarah M. Yarnell ${ }^{\mathrm{b}}$ \\ ${ }^{a}$ U.S. Geological Survey, Placer Hall, 6000 J Street, Sacramento, CA 95819, United States \\ ${ }^{\mathrm{b}}$ Department of Hydrologic Sciences, University of California, Davis, One Shields Avenue, Davis, CA 95616, United States
}

Received 19 July 2004; received in revised form 15 November 2004; accepted 19 November 2004

Available online 19 January 2005

\begin{abstract}
This study examines the development of a conceptual model of sediment processes in the upper Yuba River watershed; and we hypothesize how components of the conceptual model may be spatially distributed using a geographical information system (GIS). The conceptual model illustrates key processes controlling sediment dynamics in the upper Yuba River watershed and was tested and revised using field measurements, aerial photography, and low elevation videography. Field reconnaissance included mass wasting and channel storage inventories, assessment of annual channel change in upland tributaries, and evaluation of the relative importance of sediment sources and transport processes. Hillslope erosion rates throughout the study area are relatively low when compared to more rapidly eroding landscapes such as the Pacific Northwest and notable hillslope sediment sources include highly erodible andesitic mudflows, serpentinized ultramafics, and unvegetated hydraulic mine pits. Mass wasting dominates surface erosion on the hillslopes; however, erosion of stored channel sediment is the primary contributor to annual sediment yield. We used GIS to spatially distribute the components of the conceptual model and created hillslope erosion potential and channel storage models. The GIS models exemplify the conceptual model in that landscapes with low potential evapotranspiration, sparse vegetation, steep slopes, erodible geology and soils, and high road densities display the greatest hillslope erosion potential and channel storage increases with increasing stream order. In-channel storage in upland tributaries impacted by hydraulic mining is an exception. Reworking of stored hydraulic mining sediment in low-order tributaries continues to elevate upper Yuba River sediment yields. Finally, we propose that spatially distributing the components of a conceptual model in a GIS framework provides a guide for developing more detailed sediment budgets or numerical models making it an inexpensive way to develop a roadmap for understanding sediment dynamics at a watershed scale.

Published by Elsevier B.V.
\end{abstract}

Keywords: Yuba River; Conceptual model; Hillslope erosion; Sediment storage; Hydraulic mining

\section{Introduction}

* Corresponding author. Tel.: +1 9162783165 .

E-mail address: jacurtis@usgs.gov (J.A. Curtis).
Understanding processes that erode and route sediment in watersheds has long been of interest to

0169-555X/\$ - see front matter. Published by Elsevier B.V.

doi:10.1016/j.geomorph.2004.11.019

This article is a U.S. government work, and is not subject to copyright in the United States. 
earth scientists (Gilbert, 1917; Walling, 1983; Macklin and Lewin, 1989; Madej and Ozaki, 1996; Nelson and Booth, 2002; Kasai et al., 2004). Advances in theoretical geomorphology, computer science, remote sensing, and geographic information systems (GIS) have facilitated the development of conceptual models (see review in Shroder and Bishop, 2003). However, spatially distributing sediment processes in large high-relief watersheds can be problematic as governing factors such as topography, geology, vegetation, and runoff are highly variable over short distances.

Traditional techniques used to spatially distribute sediment processes at the watershed scale include sediment budgets, where extensive field data are collected at representative locations and extrapolated to unsampled locations (Dietrich and Dunne, 1978;
Kelsey, 1980; Trimble, 1983; Roberts and Church, 1986; Reid and Dunne, 1996) and numerical watershed sediment models (see overview in Singh, 1995). For such approaches to be successful, a necessary first step is to determine the principal factors controlling watershed sediment dynamics. For the upper Yuba River, we began this process by developing a comprehensive conceptual model of sediment sources and transport processes. Using GIS, we then hypothesize how the components of the conceptual model may be spatially distributed throughout a topographically and geologically diverse watershed.

This study is part of the California Bay-Delta Authority (CBDA) Upper Yuba River Studies Program (UYRSP, http://www.nasites.com/pam/yuba/), which is currently evaluating options for introducing spring-run Chinook salmon and steelhead trout

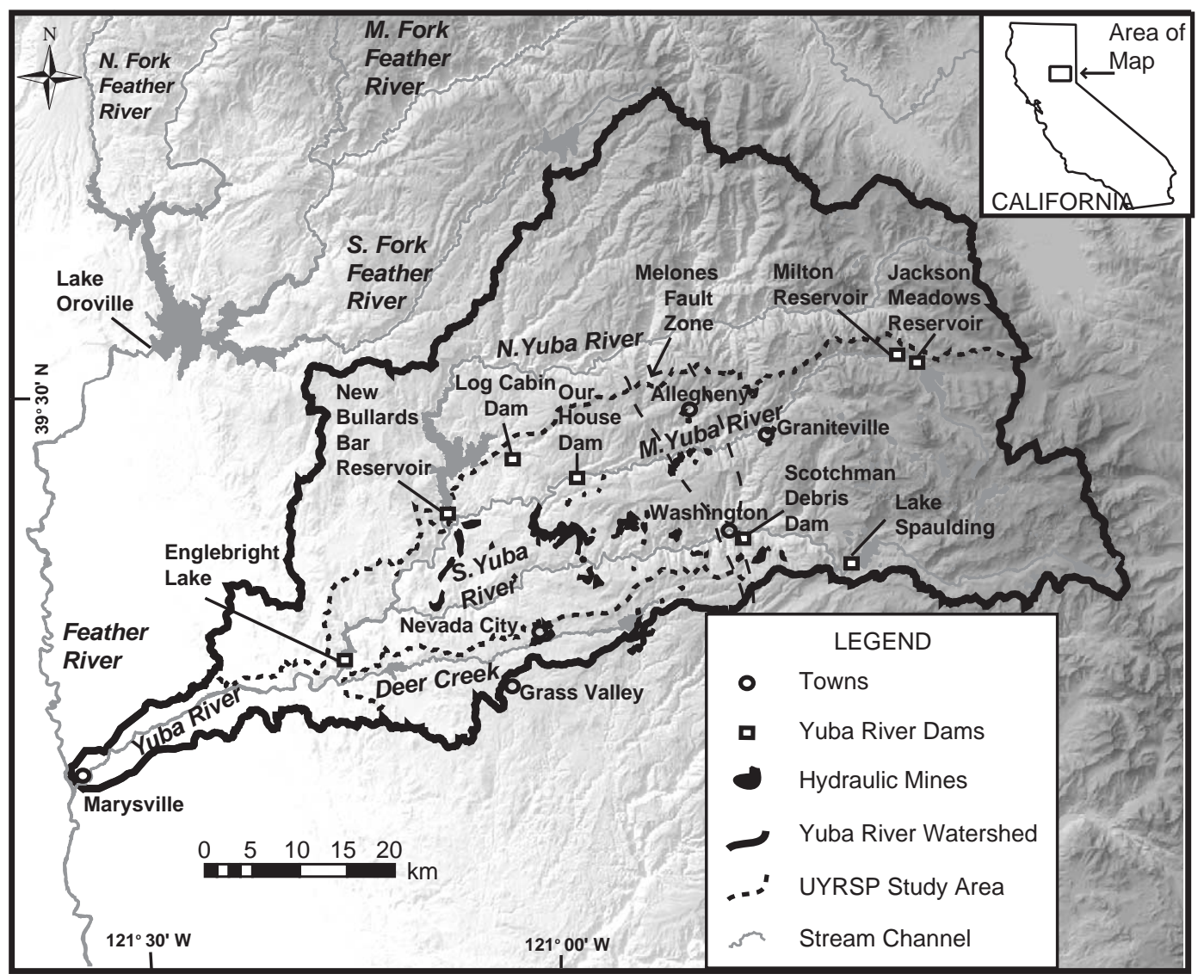

Fig. 1. Map of the Yuba River watershed showing the location of the Upper Yuba River Studies Program study area. 
upstream of Englebright Lake located in the foothills of the northwestern Sierra Nevada, CA (Fig. 1). As part of this restoration effort, process-oriented sediment studies were undertaken to support investigations of the type and quality of anadromous fish habitat and long-term viability of fish introduction strategies. During the feasibility phase of the UYRSP, the conceptual model provided a means of identifying key sediment sources, erosion and transport processes, and linkages and was further utilized to develop a watershed-scale numerical sediment-transport model (Flint et al., 2004).

\section{Study area}

The Yuba River, a tributary to the Feather River in northern California, drains $\sim 3480 \mathrm{~km}^{2}$ along the western slope of the Sierra Nevada (Fig. 1). The upper Yuba River watershed encompasses the area upstream of Englebright Lake; and the study area includes two tributaries, the Middle Yuba River (MYR) and South Yuba River (SYR). The upstream study area boundaries are Milton Reservoir on the MYR and Lake Spaulding on the SYR.

The study area has a Mediterranean climate with hot/dry summers and cool/wet winters. Vegetation ranges from oak woodlands in the lower elevations to mixed conifers in the middle and higher elevations. Soils are divided into shallow hillslope gravelly loams and deeper ridge top gravelly clay loams (Brittan, 1975; Hanes, 1994). Total mean annual precipitation ranges from $50 \mathrm{~cm}$ at Marysville at the western downstream end of the watershed to more than 150 $\mathrm{cm}$ at the eastern margin of the watershed along the Sierra Nevada crest (WRCC, 2003).

Runoff is typically generated by warm winter Pacific storms, spring snowmelt, or occasionally by convective storms generated in the late summer or early autumn by subtropical air masses from the Gulf of Mexico (Kattelman, 1996). Beginning in November, Pacific frontal systems bring winter precipitation into northern California, resulting in $\sim 85 \%$ of precipitation falling between November and April. The 1200 to $1800-\mathrm{m}$ elevation range is susceptible to rain-on-snow events (CDWR, 1966), which have the greatest magnitude, duration, and ability to mobilize sediment.

\subsection{Geologic and mining history}

Cenozoic geologic history includes uplift and tilting of the Sierra Nevada and at least two late Quaternary glaciations (Lindgren, 1911; Bateman and Wahrhaftig, 1966; Christensen, 1966; James et al., 2002). Uplift and tilting reorganized drainage networks and initiated a period of sustained channel incision. The modern Yuba River began incising $\sim 5$ Ma (Wakabayashi and Sawyer, 2001). The easternmost portion of the basin was glaciated during the Quaternary and is mantled by till and glacial outwash deposited by late Quaternary valley glaciers (James et al., 2002). The underlying bedrock geology in the study area is composed primarily of Paleozoic metasediments and metavolcanics, Paleozoic and Mesozoic granitics, and a Mesozoic ophiolite. Eocene auriferous sediments and Miocene-Pliocene rhyolites, rhyolitic sediments, and andesitic mudflows cap the ridge tops (Saucedo and Wagner, 1992).

Auriferous sediments, deposited by the ancestral Yuba River (Whitney, 1880; Lindgren, 1911; Yeend, 1974), were hydraulically mined during the California Gold Rush of the mid- to late 1800 s and during a period of protracted twentieth century licensed mining. Hydraulic mining involved directing high-pressure water cannons at Eocene gravel exposures (Fig. 2) and washing excavated sediment through mercury laden sluice boxes (Bowie, 1905; May, 1970; Averill, 1976). Tailings were ultimately conveyed into adjacent watercourses leading to substantial increases in sediment loads and downstream channel aggradation (Hall, 1880; Turner, 1891). Gilbert (1917) estimated that unlicensed hydraulic mining contributed $\sim 522$ million $\mathrm{m}^{3}$ of sediment to Yuba River channels. In 1884 , due to adverse downstream sedimentation effects, large-scale hydraulic mining was ended by court injunction (Sawyer Decision). A protracted period of licensed mining began in 1893 (Caminetti Act) and continued in the Yuba River basin until the 1950s. Licensed hydraulic mining contributed approximately 4.8 million $\mathrm{m}^{3}$ of sediment to Yuba River channels (James, submitted for publication).

Extensive remobilization of stored hydraulic mining sediment began as early as 1861 when severe winter storms delivered substantial volumes of sediment to the Central Valley. In 1941, Englebright Dam (Fig. 1) was built to trap hydraulic mining sediment 


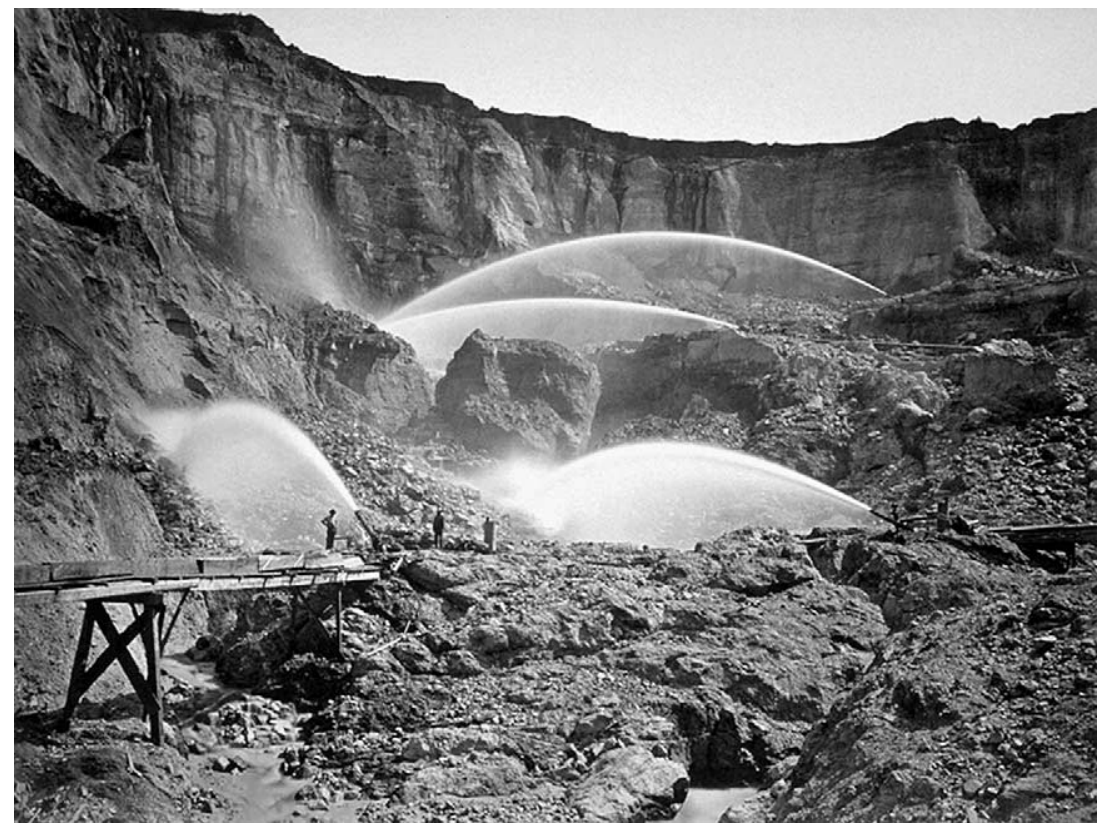

Fig. 2. Hydraulic mining at Malakoff Diggings located in the South Yuba River watershed (circa 1876). (Historic photograph taken by Carleton E. Watkins is located in the Hearst Mining Collection at the Bancroft Library, University of California at Berkeley).

generated higher in the upper Yuba River watershed. Although portions of the mainstem MYR and SYR have recovered their pre-mining bed elevations, significant volumes of hydraulic mining sediment remain stored in upland tributaries. Previous studies in the Yuba River and adjacent watersheds (Wildman, 1981; James, 1989, 1993; Curtis, 1999) indicate that low-order tributaries aggraded with vast quantities of hydraulic mining sediment are asymptotically incising to pre-mining channel bed elevations; therefore, remobilization of hydraulic mining sediment continues to affect sediment yields from impacted basins.

Two important hard rock mining districts in the study area are the Alleghany District, located within Kanaka Creek and along the MYR, and the Graniteville District located in Poorman Creek, a tributary of the SYR (Fig. 1). The volume of tailings conveyed into nearby stream channels by hard rock mining is significant, although much smaller than that contributed by hydraulic mining and difficult to quantify as there are no accurate estimates of tailings production. Gold production in the Alleghany District from 1900 to 1965 is estimated to have been more than $\$ 50$ million based on 1968 gold prices (Clark and Fuller, 1968). Using a ratio of $\$ 18.30 /$ tonnes (Ferguson and
Gannett, 1932), hard rock mining produced approximately 1 million $\mathrm{m}^{3}$ of waste rock prior to 1968 . No requirements for impounding hard rock tailings existed prior to 1912 ; therefore perhaps $50 \%$ or 0.5 million $\mathrm{m}^{3}$ was conveyed into stream channels. Although the estimate of sediment derived from hard rock mining is small compared to that produced by hydraulic mining, aggradation occurred along both Kanaka and Poorman Creeks. Currently, hard rock mines operate intermittently as the price of gold fluctuates.

\section{A conceptual model of sediment processes}

An initial conceptual model of sediment dynamics at a watershed-scale was developed for the upper Yuba River watershed using interpretations of aerial photographs (ca. 1939 and 2000), published Yuba River sediment studies (Gilbert, 1917; Yuan, 1979; Wildman, 1981; CDWR, 1987), literature describing sediment budgets in mountainous terrain (Dietrich and Dunne, 1978; Roberts and Church, 1986; Reid and Dunne, 1996), and impacts associated with hydraulic mining in adjacent watersheds (James, 1989, 1993; Curtis, 1999). Subsequent field reconnaissance and 


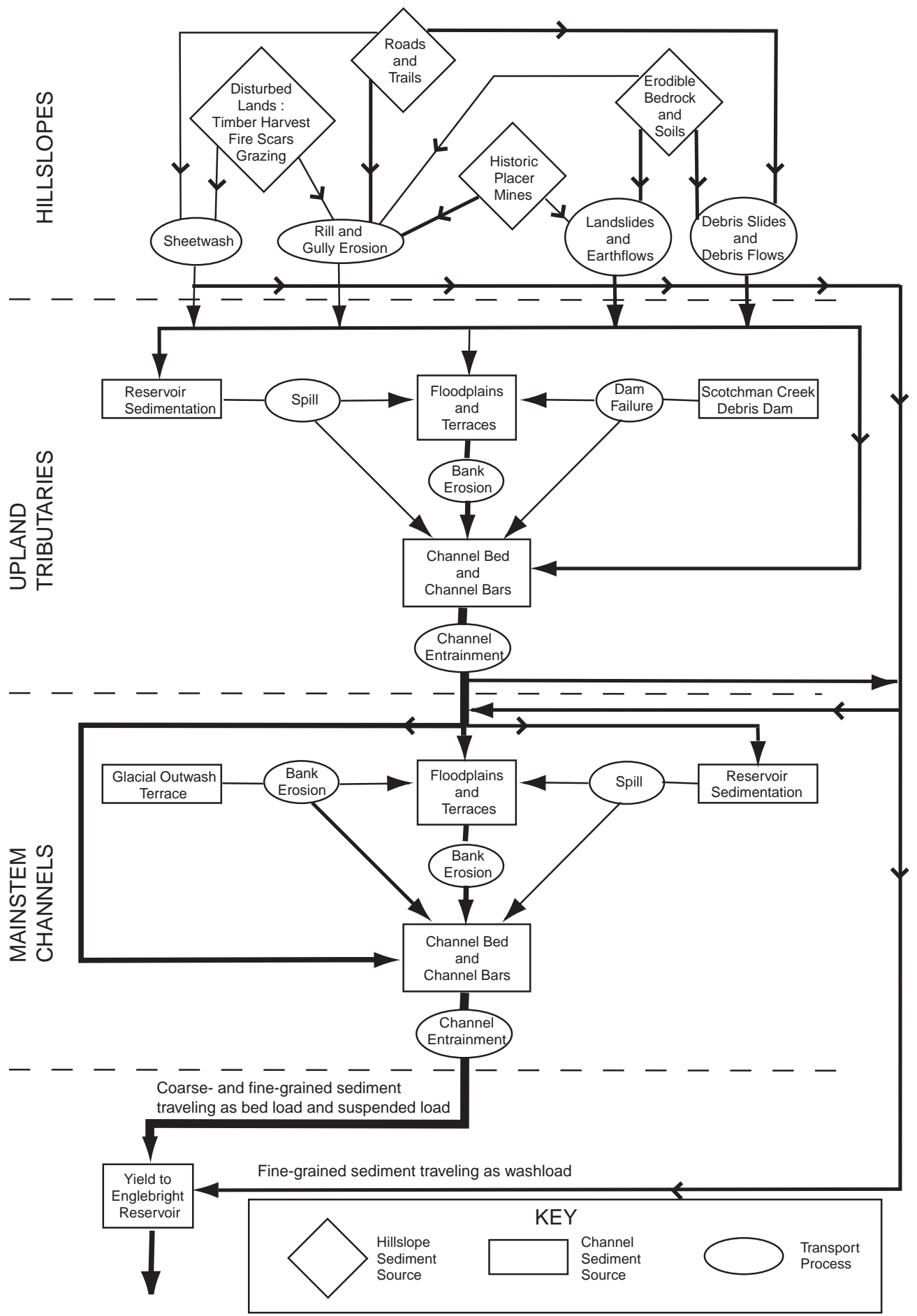

Fig. 3. A conceptual model of sediment process in the upper Yuba River watershed. Arrows denote linkages between compartments and transport directions; and line thicknesses indicate the hypothesized relative magnitude of basin-wide sediment transport. 
GIS analyses clarified the relative importance of model components and identified sediment source locations where key transport processes occur, thereby enabling verification and refinement of the model. As important sediment sources and transport processes were illuminated, components and linkages in the initial conceptual model were removed or moved; and line thicknesses indicating the relative magnitude of transport processes were defined.

The conceptual model is partitioned into three components: hillslopes, upland tributaries, and mainstem channels (Fig. 3). The three components are further compartmentalized into hillslope sediment sources, channel sediment sources, and transport processes. Arrows with variable line thicknesses denote linkages between compartments and transport directions and indicate the hypothesized relative magnitude of basin-wide sediment transport (e.g., thicker lines represent greater transport). The final conceptual model (Fig. 3) will be referred to and described throughout the paper as techniques for verifying and refining the model are discussed.

\section{Methods used to verify and refine the conceptual model and spatially distributed model components}

\subsection{Sediment source and erosion processes inventory}

Reconnaissance of the study area focused on clarifying the relative magnitudes of conceptual model compartments at the watershed scale. Field sites were selected using stratified sampling, which targeted hypothesized sediment source areas. Hillslope sources of interest included erodible bedrock lithologies, historic open pit placer mines, road prisms, and other disturbed lands (e.g., timber harvests, fire scars, and grazed lands). Channel sources included terraces, floodplains, channel bars, and debris jams in both upland tributaries and mainstem channels.

Mass wasting and surface erosion of hillslope sediment as well as channel erosion contribute to the annual sediment yield of the upper Yuba River. Mass wasting includes all soil and rock material transported downslope from gravitational forces. Conversely, surface erosion encompasses soil and rock material transported by water traveling overland to the nearest stream channel and includes sheet, rill, and gully erosion. Channel storage includes sediment deposited in a stream channel after a period of transport that is susceptible to both entrainment and cutbank erosion.

Active mass wasting sites $(n=59)$ throughout the study area were mapped on successive aerial photographs flown in June 1939 (1:22,000) and July 2000 $(1: 15,840)$. Mapping criteria included unvegetated head scarps, bare slopes, scoured stream channels, and hummocky topography. Erosion classes included debris flows, debris slides, and landslides, which were differentiated using standard terminology (CDMG, 1999). Pre-1939 landslides, which have steep unvegetated head scarps that are chronically eroded by both debris slides and debris flows, are included in a separate class. As individual processes at pre-1939 landslide sites could not be differentiated, mass wasting is reported collectively at these sites (Table 1).

Measurements collected at mass wasting field sites ( $n=22$; Fig. 4) included sediment delivery (the

Table 1

Mass wasting inventory

\begin{tabular}{|c|c|c|c|c|c|c|c|c|c|}
\hline $\begin{array}{l}\text { Erosion } \\
\text { classes }\end{array}$ & $\begin{array}{l}\text { Average } \\
\text { slope }^{a} \\
(\mathrm{~m} / \mathrm{m})\end{array}$ & $\begin{array}{l}\text { Average } \\
\text { total depth } \\
(\mathrm{m})\end{array}$ & $\begin{array}{l}\text { Average percent } \\
\text { delivered }^{\mathrm{b}}(\%)\end{array}$ & $\begin{array}{l}\text { Total sediment } \\
\text { production }\left(\mathrm{m}^{3}\right)\end{array}$ & $\begin{array}{l}\text { Percent of } \\
\text { total sediment } \\
\text { production }(\%)\end{array}$ & $\begin{array}{l}\text { Average annual } \\
\text { delivery rate } \\
\left(\mathrm{m}^{3} / \mathrm{km}^{2} / \text { year }\right)\end{array}$ & $\begin{array}{l}\text { Number } \\
\text { of sites } \\
\text { visited }\end{array}$ & $\begin{array}{l}\text { Total sites } \\
\text { number } \\
\text { of sites }\end{array}$ & $\begin{array}{l}\text { Percent } \\
\text { of total } \\
\text { sites }(\%)\end{array}$ \\
\hline Debris flows & 0.57 & 1.79 & 40 & 54,460 & 6 & 0.7 & 3 & 6 & 10 \\
\hline Debris slides & 0.58 & 0.42 & 69 & 56,020 & 6 & 0.8 & 10 & 29 & 49 \\
\hline Landslides & 0.53 & 3.33 & 40 & 178,860 & 18 & 2.4 & 3 & 3 & 5 \\
\hline $\begin{array}{l}\text { Pre-1939 } \\
\text { landslides }\end{array}$ & 0.46 & 3.40 & 49 & 676,420 & 70 & 9.2 & 6 & 21 & 36 \\
\hline Study area & & & & 965,760 & & 13.1 & 22 & 59 & \\
\hline
\end{tabular}

${ }^{\text {a }}$ Measured from USGS 1:24,000 maps.

b Represents average of field measurements for each erosion class.

c Based on 62-year interval between aerial photography flights (1939 to 2000) and $1192 \mathrm{~km}^{2}$ drainage area. 


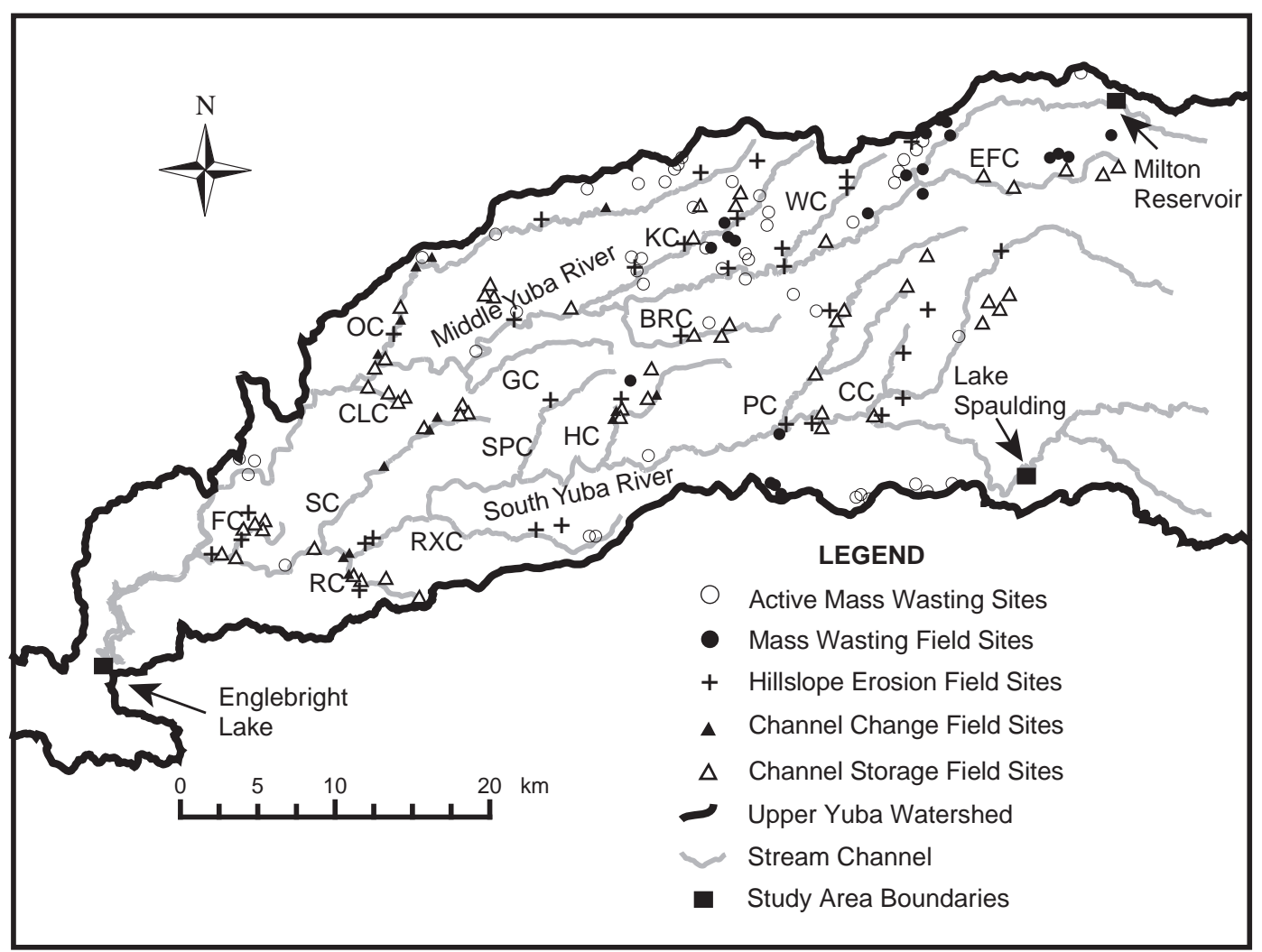

Fig. 4. Map of study area showing field sites including mass wasting sites, hillslope erosion sites, channel storage sites, and channel change sites. Abbreviations for upland tributaries labeling are FC, French Corral Creek; SC, Shady Creek; RC, Rush Creek; RXC, Rock Creek; SPC, Spring Creek; HC, Humbug Creek; PC, Poorman Creek; CC, Canyon Creek; CLC, Clear Creek; OC, Oregon Creek; GC, Grizzly Creek; BRC, Bloody Run Creek; KC, Kanaka Creek; WC, Wolf Creek; EFC, East Fork Creek.

percentage of mobilized sediment delivered to a stream channel) and post-1939 scarp areas and mean depths. Field measurements of sediment delivery and mean evacuated depth were averaged within each mass wasting class and extrapolated to unvisited sites. The total volume of sediment delivered to adjacent stream channels from each site was then calculated as

$V=D^{*} A^{*} \mathrm{SD}$

where $V$ is the total volume of sediment delivered by mass wasting from 1939 to $2000\left(\mathrm{~m}^{3}\right), D$ is the average evacuated depth (m), $A$ is the post-1939 scarp area $\left(\mathrm{m}^{2}\right)$, and SD is sediment delivery (\%).

Basin-wide mass wasting from 1939 to 2000 was estimated using aerial photography and field measurements; and the relative importance of individual mass wasting processes was determined based on the volume of sediment delivered and the frequency of occurrence on the landscape. The 1939 and 2000 aerial photographs represented the earliest and most recent aerial photography of the upper Yuba River watershed and therefore define the 62-year period of interest.

Active mass wasting was evident on the 1939 aerial photographs at all 59 sites thus complicating calculation of erosion occurring during the period of interest. However, the difference in scarp areas between the 1939 and 2000 aerial photographs was apparent at all 59 sites; and post-1939 scarp areas were estimated on the 2000 photographs to the nearest $1.0 \mathrm{~mm}^{2}\left(16 \mathrm{~m}^{2}\right.$ ground-distance $)$. Total sediment delivery from each mass wasting class was calculated and divided by 62 years to estimate an average annual sediment delivery rate for each class (Table 1). 
The upper Yuba River is a large topographically and geologically diverse basin with relatively low hillslope erosion rates; thus, quantifying surface erosion over the short project period was not attempted. However, the relative importance of hillslope source areas and erosion processes was evaluated

Table 2

Hillslope erosion data used to develop erosion potential model

\begin{tabular}{|c|c|c|c|c|c|c|c|c|c|c|c|}
\hline Site & Geology $^{a}$ & Disturbance $^{\mathrm{b}}$ & Vegetation $^{\mathrm{c}}$ & Over-story $^{\mathrm{d}}$ & Under-story $^{\mathrm{d}}$ & Grasses $^{\mathrm{d}}$ & Litter $^{\mathrm{d}}$ & $\begin{array}{l}\text { Bare } \\
\text { rock }^{\mathrm{d}}\end{array}$ & $\begin{array}{l}\text { Type of } \\
\text { hillslope } \\
\text { erosion }\end{array}$ & $\begin{array}{l}\text { Severity of } \\
\text { hillslope } \\
\text { erosion }(0-3)^{\mathrm{f}}\end{array}$ & $\begin{array}{l}\text { Road- related } \\
\text { erosion }(0-3)^{f}\end{array}$ \\
\hline 1 & grMz & M & $\mathrm{C} / \mathrm{D}$ & 1 & 2 & 1 & 5 & 1 & None & 0 & 0 \\
\hline 2 & $\mathrm{Pz}$ & $\mathrm{M}, \mathrm{R}, \mathrm{G}$ & $\mathrm{C}$ & 1 & 3 & 1 & 1 & 2 & $\mathrm{R}, \mathrm{G}, \mathrm{MW}$ & 1 & 1 \\
\hline 3 & $\mathrm{Pz}$ & $\mathrm{R}$ & $\mathrm{C}$ & 1 & 1 & 1 & 1 & 5 & MW & 3 & 1 \\
\hline 4 & $\mathrm{Pz}$ & $\mathrm{R}$ & LO & 4 & 2 & 1 & 4 & 1 & MW & 1 & 2 \\
\hline 5 & $\mathrm{Pz}$ & $\mathrm{M}, \mathrm{R}$ & LO & 3 & 1 & 1 & 2 & 2 & MW & 1 & 1 \\
\hline 6 & Tvp & $\mathrm{R}$ & $\mathrm{C} / \mathrm{D}$ & 4 & 1 & 1 & 5 & 1 & None & 0 & 1 \\
\hline 7 & Tvp & None & $\mathrm{C}$ & 1 & 2 & 1 & 1 & 2 & MW & 2 & 0 \\
\hline 8 & $\mathrm{Pz}$ & None & LO & 1 & 1 & 2 & 1 & 2 & MW & 1 & 0 \\
\hline 9 & $\mathrm{mv}$ & $\mathrm{M}, \mathrm{R}$ & $\mathrm{C} / \mathrm{D}$ & 3 & 2 & 1 & 4 & 1 & None & 0 & 1 \\
\hline 10 & um & None & $\mathrm{C} / \mathrm{D}$ & 2 & 1 & 1 & 5 & 1 & None & 0 & 0 \\
\hline 11 & um & $\mathrm{M}, \mathrm{R}$ & $\mathrm{C} / \mathrm{D}$ & 1 & 1 & 1 & 1 & 5 & None & 0 & 2 \\
\hline 12 & $\mathrm{gb}$ & $\mathrm{R}$ & LO & 1 & 1 & 1 & 1 & 4 & R,G,MW & 1 & 1 \\
\hline 13 & $\mathrm{gb}$ & None & LO & 2 & 3 & 1 & 1 & 2 & None & 0 & 0 \\
\hline 14 & $\mathrm{gb}$ & None & LO & 5 & 1 & 2 & 3 & 1 & None & 0 & 0 \\
\hline 15 & um & $\mathrm{R}$ & $\mathrm{C}$ & 1 & 2 & 1 & 1 & 3 & None & 0 & 3 \\
\hline 16 & um & $\mathrm{T}, \mathrm{R}$ & $\mathrm{C}$ & 4 & 1 & 1 & 2 & 1 & G,MW & 3 & 2 \\
\hline 17 & $\mathrm{Pz}$ & M & $\mathrm{C} / \mathrm{D}$ & 3 & 2 & 1 & 2 & 2 & MW & 2 & 0 \\
\hline 18 & Tvp & $\mathrm{T}, \mathrm{R}, \mathrm{G}$ & $\mathrm{C}$ & 3 & 1 & 1 & 5 & 1 & None & 0 & 1 \\
\hline 19 & Tvp & $\mathrm{T}, \mathrm{R}$ & $\mathrm{O}$ & 1 & 5 & 1 & 5 & 1 & None & 0 & 1 \\
\hline 20 & $\mathrm{Pz}$ & None & $\mathrm{C} / \mathrm{D}$ & 5 & 1 & 1 & 3 & 2 & None & 0 & 0 \\
\hline 21 & grMz & $\mathrm{R}$ & $\mathrm{C}$ & 2 & 2 & 1 & 1 & 2 & None & 0 & 1 \\
\hline 22 & $\mathrm{Pz}$ & $\mathrm{T}, \mathrm{R}, \mathrm{F}$ & $\mathrm{C}$ & 2 & 1 & 1 & 2 & 1 & $\mathrm{R}$ & 1 & 2 \\
\hline 23 & $\mathrm{Pz}$ & $\mathrm{M}, \mathrm{R}$ & $\mathrm{C} / \mathrm{D}$ & 4 & 2 & 1 & 2 & 1 & None & 0 & 1 \\
\hline 24 & grMz & $\mathrm{T}$ & $\mathrm{C}$ & 3 & 4 & 1 & 5 & 1 & None & 0 & 0 \\
\hline 25 & $\mathrm{Pz}$ & $\mathrm{R}$ & $\mathrm{C}$ & 4 & 2 & 1 & 4 & 1 & MW & 1 & 1 \\
\hline 26 & um & $\mathrm{M}, \mathrm{R}$ & $\mathrm{C}$ & 3 & 2 & 1 & 1 & 1 & MW & 1 & 1 \\
\hline 27 & $\mathrm{Pz}$ & M & $\mathrm{C} / \mathrm{D}$ & 4 & 2 & 1 & 4 & 1 & MW & 2 & 0 \\
\hline 28 & Tvp & $\mathrm{R}$ & $\mathrm{C} / \mathrm{D}$ & 4 & 4 & 1 & 5 & 1 & None & 0 & 1 \\
\hline 29 & $\mathrm{~J}$ & $\mathrm{R}$ & $\mathrm{M}$ & 1 & 1 & 4 & 1 & 1 & None & 0 & 1 \\
\hline 30 & grMz & $\mathrm{T}, \mathrm{R}$ & $\mathrm{C} / \mathrm{D}$ & 5 & 1 & 1 & 2 & 1 & MW & 1 & 1 \\
\hline 31 & grMz & $\mathrm{M}, \mathrm{R}, \mathrm{F}$ & LO & 2 & 1 & 5 & 2 & 1 & None & 0 & 1 \\
\hline 32 & grMz & $\mathrm{M}, \mathrm{R}, \mathrm{G}$ & LO & 2 & 1 & 5 & 1 & 1 & None & 0 & 1 \\
\hline 33 & grMz & $\mathrm{M}, \mathrm{R}, \mathrm{F}$ & LO & 3 & 1 & 4 & 1 & 2 & MW & 2 & 1 \\
\hline 34 & grMz & None & $\mathrm{C} / \mathrm{D}$ & 4 & 3 & 1 & 4 & 1 & $\mathrm{R}$ & 1 & 0 \\
\hline 35 & $\mathrm{grMz}$ & $\mathrm{R}$ & $\mathrm{C} / \mathrm{D}$ & 3 & 2 & 2 & 4 & 1 & MW & 1 & 2 \\
\hline 36 & Tvp & $\mathrm{T}, \mathrm{R}, \mathrm{G}, \mathrm{F}$ & $\mathrm{C} / \mathrm{D}$ & 1 & 1 & 1 & 5 & 1 & None & 0 & 2 \\
\hline 37 & Tvp & None & $\mathrm{C} / \mathrm{D}$ & 4 & 2 & 1 & 5 & 1 & None & 0 & 1 \\
\hline 38 & $\mathrm{grMz}$ & $\mathrm{R}$ & $\mathrm{C}$ & 1 & 1 & 1 & 2 & 2 & None & 0 & 1 \\
\hline 39 & grMz & $\mathrm{T}, \mathrm{R}$ & $\mathrm{C}$ & 2 & 1 & 1 & 4 & 1 & None & 0 & 1 \\
\hline
\end{tabular}

${ }^{a}$ Jurassic diorite (J), Mesozoic granitics (grMz), metavolcanics (mv), Paleozoic metasediment (Pz), gabbro (gb), Tertiary (Miocene-Plioene) volcanics-andesitic mudflows (Tvp), serpentinized ultramafic rocks (um).

${ }^{b}$ Timber harvesting $(T)$, mining $(M)$, roads $(R)$, grazing $(G)$, fire $(F)$.

${ }^{c}$ Conifer forest (C), deciduous oak woodland (D), conifer/deciduous woodland (C/D), live oak woodland (LO), meadow/grassland (M), other including manzanita $(\mathrm{O})$.

d $1=0-20 \%, 2=20-40 \%, 3=40-60 \%, 4=60-80 \%, 5=80-100 \%$.

e Rilling (R), gullying (G), mass wasting (MW).

f $0=$ negligible, $1=$ minor, $2=$ moderate, $3=$ severe. 
Table 3

Storage element stability classes ${ }^{\mathrm{a}}$

\begin{tabular}{ll}
\hline Stability class & Description \\
\hline Active & $\begin{array}{l}\text { Moves at least once every few years. } \\
\text { Semi-active }\end{array}$ \\
Inactive & $\begin{array}{l}\text { moved every 5-20 years. } \\
\text { Moves only during extreme events } \\
\text { every 20-100 years and becomes } \\
\text { well-vegetated in the interim. } \\
\text { Deposits are not accumulating under } \\
\text { present climate or channel regime but } \\
\text { may be susceptible to cutbank erosion. }\end{array}$ \\
\end{tabular}

${ }^{\mathrm{a}}$ Kelsey et al. (1987).

at 39 hillslope sites (Fig. 4). Field reconnaissance at these locations included documenting the underlying bedrock geology, vegetation type and percent cover, and all land-use disturbances. In addition, the type and relative severity of hillslope and road-related erosion was assessed (Table 2).

Channel storage was assessed along 56 channel reaches selected from a stream network map. Study reaches varied in length and included zero through fifth-order channels. The volume of channel storage above the 2002 thalweg within each study reach was measured using a stadia rod and tape measure. The length, width, and height above the 2002 thalweg were measured for discrete storage elements, including debris jams, channel bars, floodplains, and terraces. The stability of each storage element was assessed based on grain size, location relative to the channel thalweg, and age and type of vegetation (Table 3). Average storage volumes were calculated for zero through fifth-order channels using the field inventory data and are reported as a volume per unit channel length $\left(\mathrm{m}^{3} / \mathrm{m}\right)$, which enabled comparison among study sites (Table 4).

The relative importance of upland tributary and mainstem channel erosion processes were further evaluated using low elevation aerial videography of the mainstem channels (Barclay, 2002) and estimates of channel change in upland tributaries. The low elevation aerial video enabled an assessment of important channel storage sites throughout the MYR and SYR mainstem channels, which could not be evaluated using available large-scale $(1: 15,840)$ aerial photography. Evaluation of channel change in four upland tributaries enabled an assessment of the relative magnitudes of cutbank erosion and entrainment. Beginning in 1998, three channel reaches were selected in Oregon Creek, Rush Creek, Humbug Creek, and Shady Creek (Fig. 4) and cross-sections were measured within each reach. The cross-sections were then re-measured one to three times from 1999 to 2003 allowing estimates of annual channel change (Table 5).

Table 4

Inventory of channel storage above the 2002 thalweg

\begin{tabular}{|c|c|c|c|c|c|c|c|c|c|c|}
\hline $\begin{array}{l}\text { Strahler } \\
\text { stream } \\
\text { order }\end{array}$ & $\begin{array}{l}\text { Average } \\
\text { drainage } \\
\text { area } \\
\left(\mathrm{km}^{2}\right)\end{array}$ & $\begin{array}{l}\text { Average } \\
\text { length } \\
\text { of study } \\
\text { reach }(\mathrm{m})\end{array}$ & $\begin{array}{l}\text { Average } \\
\text { study } \\
\text { reach } \\
\text { slope } \\
(\mathrm{m} / \mathrm{m})\end{array}$ & $\begin{array}{l}\text { Average } \\
\text { storage per } \\
\text { unit channel } \\
\text { length } \\
\left(\mathrm{m}^{3} / \mathrm{m}\right)\end{array}$ & $\begin{array}{l}\text { Reaches } \\
\text { with stored } \\
\text { hydraulic } \\
\text { mining and } \\
\text { glacial } \\
\text { sediment } \\
\left(\mathrm{m}^{3} / \mathrm{m}\right)\end{array}$ & $\begin{array}{l}\text { Cumulative } \\
\text { study area } \\
\text { channel } \\
\text { length }(\mathrm{km})\end{array}$ & $\begin{array}{l}\text { Basin-wide } \\
\text { sediment } \\
\text { storage } \\
\left(\mathrm{m}^{3} \times 10^{6}\right)\end{array}$ & $\begin{array}{l}\text { Sediment } \\
\text { storage in } \\
\text { discrete } \\
\text { storage } \\
\text { elements } \\
(\%)^{\mathrm{a}}\end{array}$ & $\begin{array}{l}\text { Basin-wide } \\
\text { sediment } \\
\text { storage within } \\
\text { the active } \\
\text { channel } \\
\left(\mathrm{m}^{3} \times 10^{6}\right)\end{array}$ & $\begin{array}{l}\text { Sediment } \\
\text { Storage } \\
\text { within the } \\
\text { active } \\
\text { channel } \\
(\%)^{\mathrm{b}}\end{array}$ \\
\hline Zero $(n=11)$ & 0.2 & 20 & 0.18 & 0.2 & No data & 1345 & 0.2 & $(50,30,20,0)$ & 0.23 & $(50,30,20)$ \\
\hline First $(n=11)$ & 0.8 & 30 & 0.14 & 13 & No data & 589 & 7.8 & $(1,5,1,93)$ & 0.52 & $(9,79,12)$ \\
\hline Second $(n=12)$ & 3.8 & 50 & 0.09 & 6.3 & $378^{\mathrm{c}}$ & 276 & 1.7 & $(1,15,22,62)$ & 0.66 & $(3,39,58)$ \\
\hline Third $(n=11)$ & 21 & 70 & 0.07 & 15 & $676^{\mathrm{c}}$ & 153 & 2.3 & $(0,8,22,70)$ & 0.69 & $(0,26,74)$ \\
\hline Fourth $(n=6)$ & 92 & 120 & 0.04 & 70 & $560^{\mathrm{c}}$ & 60 & 4.2 & $(0,5,5,90)$ & 0.40 & $(1,47,52)$ \\
\hline Fifth $^{\mathrm{d}}(n=9)$ & 730 & 190 & 0.02 & 348 & $2920^{\mathrm{e}}$ & 131 & 482 & $(0,8,12,80)$ & 9.00 & $(0,40,60)$ \\
\hline
\end{tabular}

${ }^{\mathrm{a}}$ (Debris jam \%, channel bar \%, floodplain \%, terraces \%).

b (Debris jam \%, channel bar \%, floodplain \%).

${ }^{c}$ Reaches impacted by hydraulic mining in Shady Creek.

${ }^{d}$ Fifth-order basins include the MYR and SYR.

e Glacial outwash terrace preserved on mainstem SYR. 
Table 5

Channel changes in upland tributaries from 1998 to 2003

\begin{tabular}{|c|c|c|c|c|c|c|c|}
\hline Cross-section & Drainage area $\left(\mathrm{km}^{2}\right)$ & Survey dates & $\begin{array}{l}\text { Scour } \\
\left(\mathrm{m}^{2}\right)\end{array}$ & $\begin{array}{l}\text { Fill } \\
\left(\mathrm{m}^{2}\right)\end{array}$ & $\begin{array}{l}\text { Net } \\
\text { change } \\
\left(\mathrm{m}^{2}\right)\end{array}$ & $\begin{array}{l}\text { Duration } \\
\text { between } \\
\text { surveys (days) }\end{array}$ & $\begin{array}{l}\text { Rate of annual } \\
\text { cross-sectional area } \\
\text { change }\left(\mathrm{m}^{2} / \text { year }\right)\end{array}$ \\
\hline Shady Creek DTS & 1.7 & $\begin{array}{l}9 / 7 / 98 \text { to } 7 / 7 / 99 \\
7 / 7 / 99 \text { to } 9 / 4 / 01 \\
9 / 4 / 01 \text { to } 10 / 11 / 02 \\
10 / 11 / 02 \text { to } 9 / 16 / 03 \\
\text { Net }^{\mathrm{a}} \\
\text { Average }^{\text {a }}\end{array}$ & $\begin{array}{r}-5.9 \\
-22.5 \\
-0.8 \\
-0.9 \\
-30.0\end{array}$ & $\begin{array}{l}1.6 \\
0.1 \\
1.6 \\
0.3 \\
3.6\end{array}$ & $\begin{array}{r}-4.3 \\
-22.4 \\
0.9 \\
-0.6 \\
-26.4\end{array}$ & $\begin{array}{r}302 \\
813 \\
402 \\
341 \\
1858\end{array}$ & $\begin{array}{r}-5.2 \\
-10.0 \\
0.8 \\
-0.6 \\
-3.8\end{array}$ \\
\hline Shady Creek Rust Pit & 14.0 & $\begin{array}{l}8 / 27 / 98 \text { to } 8 / 28 / 01 \\
8 / 28 / 01 \text { to } 11 / 19 / 02 \\
11 / 19 / 02 \text { to } 9 / 16 / 03 \\
\text { Net }^{\mathrm{a}} \\
\text { Average }^{\text {a }}\end{array}$ & $\begin{array}{r}-32.7 \\
-1.1 \\
-3.2 \\
-37.0\end{array}$ & $\begin{array}{l}0 \\
0.2 \\
0.9 \\
1.0\end{array}$ & $\begin{array}{r}-32.7 \\
-0.9 \\
-2.3 \\
-35.9\end{array}$ & $\begin{array}{r}1096 \\
448 \\
301 \\
1845\end{array}$ & $\begin{array}{r}-10.9 \\
-0.8 \\
-2.8 \\
-4.8\end{array}$ \\
\hline Shady Creek Shady Road & 22.7 & $\begin{array}{l}9 / 8 / 98 \text { to } 8 / 22 / 01 \\
8 / 22 / 01 \text { to } 10 / 1 / 02 \\
10 / 1 / 02 \text { to } 9 / 16 / 03 \\
\text { Net }^{a} \\
\text { Average }^{a}\end{array}$ & $\begin{array}{r}-51.1 \\
-1.5 \\
-9.7 \\
-62.3\end{array}$ & $\begin{array}{l}0.1 \\
0 \\
0 \\
0.1\end{array}$ & $\begin{array}{r}-51.0 \\
-1.5 \\
-9.7 \\
-62.2\end{array}$ & $\begin{array}{r}1056 \\
404 \\
351 \\
1811\end{array}$ & $\begin{array}{r}-17.6 \\
-1.4 \\
-10.1 \\
-9.7\end{array}$ \\
\hline Rush Creek Road Jumble ${ }^{\mathrm{b}}$ & 14.6 & $\begin{array}{l}9 / 12 / 00 \text { to } 9 / 12 / 01 \\
9 / 12 / 01 \text { to } 10 / 15 / 02 \\
\text { Net }^{\mathrm{a}} \\
\text { Average }^{\mathrm{a}}\end{array}$ & $\begin{array}{c}0 \\
-0.1 \\
-0.1\end{array}$ & $\begin{array}{l}0.3 \\
0.4 \\
0.7\end{array}$ & $\begin{array}{l}0.3 \\
0.3 \\
0.6\end{array}$ & $\begin{array}{l}365 \\
398 \\
763\end{array}$ & $\begin{array}{l}0.3 \\
0.3 \\
0.3\end{array}$ \\
\hline Rush Creek Aarons Pools ${ }^{\mathrm{b}}$ & 14.6 & $9 / 13 / 01$ to $10 / 15 / 02$ & -0.7 & 0.7 & -0.1 & 397 & -0.1 \\
\hline Rush Creek Bare Rock & 12.8 & $9 / 19 / 01$ to $8 / 9 / 02$ & -0.1 & 0.2 & 0.1 & 323 & 0.1 \\
\hline Humbug Creek Blair Pond & 10.9 & $8 / 27 / 01$ to $8 / 12 / 02$ & -0.2 & 0 & -0.2 & 350 & -0.3 \\
\hline Humbug Creek Gage & 20.9 & $\begin{array}{l}4 / 23 / 99 \text { to } 8 / 15 / 01 \\
8 / 15 / 01 \text { to } 8 / 22 / 02 \\
\text { Net }^{\mathrm{a}} \\
\text { Average }^{\mathrm{a}}\end{array}$ & $\begin{array}{l}-0.2 \\
-0.1 \\
-0.3\end{array}$ & $\begin{array}{l}0 \\
0 \\
0\end{array}$ & $\begin{array}{l}-0.2 \\
-0.1 \\
-0.3\end{array}$ & $\begin{array}{r}844 \\
372 \\
1216\end{array}$ & $\begin{array}{l}-0.1 \\
-0.1 \\
-0.1\end{array}$ \\
\hline Humbug Creek Picnic Bar & 21.3 & $\begin{array}{l}12 / 17 / 98 \text { to } 8 / 20 / 01 \\
8 / 20 / 01 \text { to } 8 / 22 / 02 \\
\text { Net }^{\mathrm{a}} \\
\text { Average }^{\mathrm{a}}\end{array}$ & $\begin{array}{l}0 \\
0 \\
0\end{array}$ & $\begin{array}{l}0 \\
0.1 \\
0.1\end{array}$ & $\begin{array}{l}0 \\
0.1 \\
0.1\end{array}$ & $\begin{array}{r}979 \\
404 \\
1383\end{array}$ & $\begin{array}{l}0 \\
0.1 \\
0.1\end{array}$ \\
\hline Oregon Creek Celestial Pools & 85.4 & $11 / 10 / 01$ to $11 / 15 / 02$ & 0 & 0.5 & 0.5 & 370 & 0.5 \\
\hline Oregon Creek Gage Pools & 56.5 & $11 / 27 / 01$ to $8 / 8 / 02$ & -0.5 & 1.6 & 1.1 & 255 & 1.6 \\
\hline Oregon Creek Road & 27.7 & $11 / 11 / 01$ to $8 / 21 / 02$ & 0 & 0 & 0 & 284 & 0 \\
\hline
\end{tabular}

${ }^{\text {a }}$ Net and average changes to cross-sections estimated for sites with three or more channel surveys.

b Rush Creek Road Jumble site relocated in 2001 to Rush Creek Aarons Pools.

\subsection{GIS analyses}

Using GIS, the channel storage and hillslope components of the conceptual model were spatially distributed resulting in an estimate of basin-wide channel storage and a hillslope erosion potential map (Fig. 5). Distributing channel storage was a relatively simple process. The arithmetic average of storage volumes and the distribution of sediment among individual storage elements (i.e., debris jams, bars, floodplains, and terraces) were calculated for each stream order class and then multiplied by cumulative channel lengths to provide basin-wide estimates (Table 3). Development of a hillslope erosion potential map warrants a more detailed explanation.

The first step was to develop a matrix of landscape attributes governing hillslope erosion processes with scaling factors and relative multipliers (Table 6) assigned based on field reconnaissance. The scaling factors signify the range of values associated with each landscape attribute and the multipliers indicate 


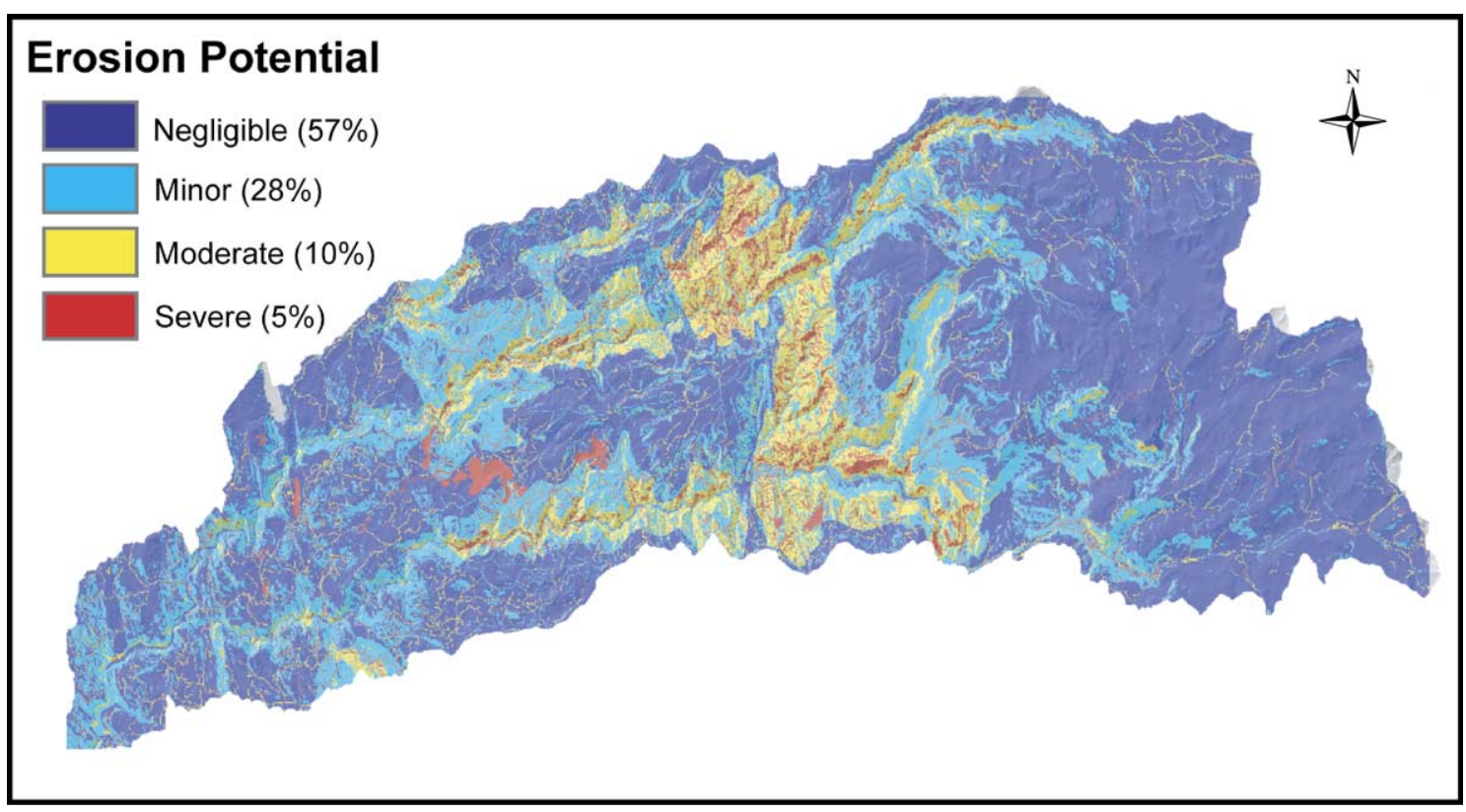

Fig. 5. Hillslope erosion potential map generated using GIS calculations. The relative erodibility classes approximate field data such that $\sim 5 \%$ of upper Yuba River hillslope are highly erodible, 10\% are moderately erodible, 28\% are slightly erodible, and 57\% are susceptible to negligible erosion.

the comparative importance. GIS data sets used in this analysis were derived from a variety of sources and include (i) soil erodibility index (kfactor; USDANRCS, 1994); (ii) Tahoe National Forest road network including road type; (iii) bedrock geology (1:250,000 scale); (iv) vegetation type (Davis and Stoms, 1996) with associated vegetation cover percentages estimated using field data; (v) slope and elevation data derived from a 30-m digital elevation model (DEM); (vi) potential evapotranspiration (PET) for the month of April calculated using a solar radiation model that relies on the DEM (Flint and Childs, 1987); (vii) digitized historic placer mine locations (Yeend, 1974); and (viii) mass wasting sites that were mapped and digitized as part of this study. GIS coverages were converted to $30-\mathrm{m}$ grids, and a calculation of hillslope erosion potential was developed that accounts for all contributing factors.

The percent vegetation cover grid did not require a scaling factor as the values ranged from 0 to 1 . Mass wasting sites and placer mines are point locations; therefore, scaling factors were either 1.0 (present) or 0 (not present). Soil erodibility values ( 0.01 to 0.44$)$, PET values (30 to $180 \mathrm{~mm} / \mathrm{month}$ ), and slope values ( 0 to
$200 \%$ ) were scaled to 1.0 . Roads were classified using the U.S. Forest Service equivalent roaded area (ERA) index (Costick, 1996), which relates road use intensity to expected erosion impacts. ERA values (4.4 to 8.25) were also scaled to 1.0. The geology scaling factors were based on field reconnaissance of factors governing the resistance to erosion, such as the presence of joints or other partings, weathering, and permeability (Selby, 1993). Elevation scaling factors were assigned based on precipitation type (i.e., snow, rain on snow, rainfall) and related runoff potential (CDWR, 1966). Elevations above $1800 \mathrm{~m}$ are largely covered by snow pack during the winter runoff season; however the 1200 - to 1800 -m zone is susceptible to rain on snow events, which can generate widespread runoff and erosion. During average precipitation conditions, elevations below $1200 \mathrm{~m}$ typically receive moderate amounts of rainfall.

Using GIS $30-\mathrm{m}$ grid coverages, representing the landscape attributes of interest, were assigned scaling factors or scaled to represent ranges between 0 and 1 (Table 6). The resultant scaled grids were then multiplied by relative weighting factors (Table 6). The final calculation produced a hillslope erosion 
Table 6

Scaling factors and relative multipliers used to estimate hillslope erosion potential

\begin{tabular}{|c|c|c|c|}
\hline $\begin{array}{l}\text { Landscape } \\
\text { attributes }\end{array}$ & Range of values & $\begin{array}{l}\text { Scaling } \\
\text { factors }\end{array}$ & $\begin{array}{l}\text { Relative } \\
\text { multiplie }\end{array}$ \\
\hline Slope & $0-200 \%$ & $0-1.0$ & 9 \\
\hline \multirow[t]{3}{*}{ Geology ${ }^{a}$} & $\begin{array}{l}\mathrm{J}, \mathrm{Q}, \mathrm{Qg}, \mathrm{grMz} \\
\mathrm{Tv}, \mathrm{mv}, \mathrm{Qv}\end{array}$ & 0.3 & 9 \\
\hline & $\mathrm{Pz}, \mathrm{gb}, \mathrm{QPc}$ & 0.5 & \\
\hline & Tvp, Ec, um & 1.0 & \\
\hline \multirow[t]{3}{*}{ Elevation } & $>1800 \mathrm{~m}$ & 0.66 & 6 \\
\hline & $1200-1800 \mathrm{~m}$ & 1.0 & \\
\hline & $<1200 \mathrm{~m}$ & 0.8 & \\
\hline $\begin{array}{l}\text { Soil erodibility } \\
\text { index (kfactor) }\end{array}$ & $0.01-0.44$ & $0-1.0$ & 9 \\
\hline $\begin{array}{l}\text { Total potential } \\
\text { evapotranspiration } \\
\text { for month of } \\
\text { April (PET) }\end{array}$ & $30-180 \mathrm{~mm} / \mathrm{month}$ & $0-1.0$ & 4 \\
\hline Vegetation cover & $0-100 \%$ & $0-1.0$ & 6 \\
\hline \multirow[t]{4}{*}{ Roads } & Paved & 0.20 & 10 \\
\hline & Gravel & 0.56 & \\
\hline & Dirt and trails & 0.66 & \\
\hline & 4 wheel drive & 1.0 & \\
\hline \multirow{2}{*}{$\begin{array}{l}\text { Historic hydraulic } \\
\text { mine pits }\end{array}$} & Present & 1.0 & 10 \\
\hline & Not present & 0 & \\
\hline \multirow[t]{2}{*}{ Mass wasting sites } & Present & 1.0 & 10 \\
\hline & Not present & 0 & \\
\hline
\end{tabular}

${ }^{\mathrm{a}}$ Explanation of geologic units: Jurassic diorite (J), Quaternary alluvium (Q), Quaternary glacial (Qg), Mesozoic granitics (grMz), Tertiary volcanics $(\mathrm{Tv})$, metavolcanics $(\mathrm{mv})$, Quaternary volcanics $(\mathrm{Qv})$, Paleozoic metasediments (Pz), gabbro (gb), QuaternaryPliocene colluvium (QPc), Tertiary (Miocene-Pliocene) volcanics (Tvp), Eocene gravels (Ec), serpentinized ultramafics (um).

potential map (Fig. 5) that was additive, where hillslope erosion potential equals

$$
\begin{aligned}
& {[\text { elevation*6] }+[(\text { slope } * \text { geology }+ \text { kfactor }) * 9]} \\
& \quad+[(\text { roads }+ \text { mines }+ \text { mass wasting sites }) * 10] \\
& \quad-\left[\text { PET }^{*} 4\right]-[\text { vegetation cover*6 }]
\end{aligned}
$$

\section{Results}

\subsection{Hillslope sediment sources}

Field reconnaissance of hillslope sediment sources indicates low hillslope erosion rates throughout the study area. Moreover, mass wasting dominates surface erosion and based on GIS calculations $95 \%$ of the upper Yuba River watershed exhibits negligible to moderate hillslope erosion potentials.

Total sediment production in the upper Yuba River from 1939 to 2000 attributable to mass wasting is $\sim 0.97$ million $\mathrm{m}^{3}$ or 2.56 million tonnes based on a conversion factor of 2.65 tonnes $/ \mathrm{m}^{3}$. Based on a 62year interval, this equates to an average annual rate of $15,600 \mathrm{~m}^{3} /$ year or $13.1 \mathrm{~m}^{3} / \mathrm{km}^{2} /$ year, when normalized by a contributing drainage area of $1192 \mathrm{~km}^{2}$. Debris slides occur most often on the landscape at $49 \%$ of the mass wasting sites; however, these sites contributed only $6 \%$ of the total sediment produced by mass wasting (Table 1). In comparison, chronic erosion of pre-1939 landslide scarps, observed at $36 \%$ of the sites, produced $70 \%$ of the sediment attributable to mass wasting.

Evidence of active hillslope erosion (rilling, gullying, and mass wasting) was documented at $44 \%$ of the 39 hillslope sites. Five percent of the hillslope sites were severely eroded, $10 \%$ moderately eroded, $28 \%$ displayed minor erosion, and $57 \%$ showed negligible erosion (Table 2). Mass wasting was documented at $88 \%$ of the eroded sites and dominates surface erosion, which was observed at $41 \%$ of the eroded sites. Road-related erosion was assessed at 28 sites; $4 \%$ of these sites were severely eroded, $21 \%$ were moderately eroded, and $75 \%$ showed minor erosion.

GIS was used to spatially distribute hillslope erosion potential. The relative erodibility classes shown in Fig. 5 approximate field data such that $\sim 5 \%$ of upper Yuba River hillslopes are highly erodible, $10 \%$ are moderately erodible, $28 \%$ are slightly erodible, and $57 \%$ are susceptible to negligible erosion. The erosion potential map further indicates that the central portion of the upper Yuba River watershed is at greater risk for hillslope erosion attributable to a combination of steep slopes, erodible soils and bedrock, and historic mining.

\subsection{In-channel storage}

A significant proportion of in-channel storage in the upper Yuba River is associated with historic hydraulic mining activities. Using white quartz pebble counts James (submitted for publication) concluded that the majority of fine (16 to $32 \mathrm{~mm}$ ) bed material, in South Yuba River mainstem and tributary channels, is 

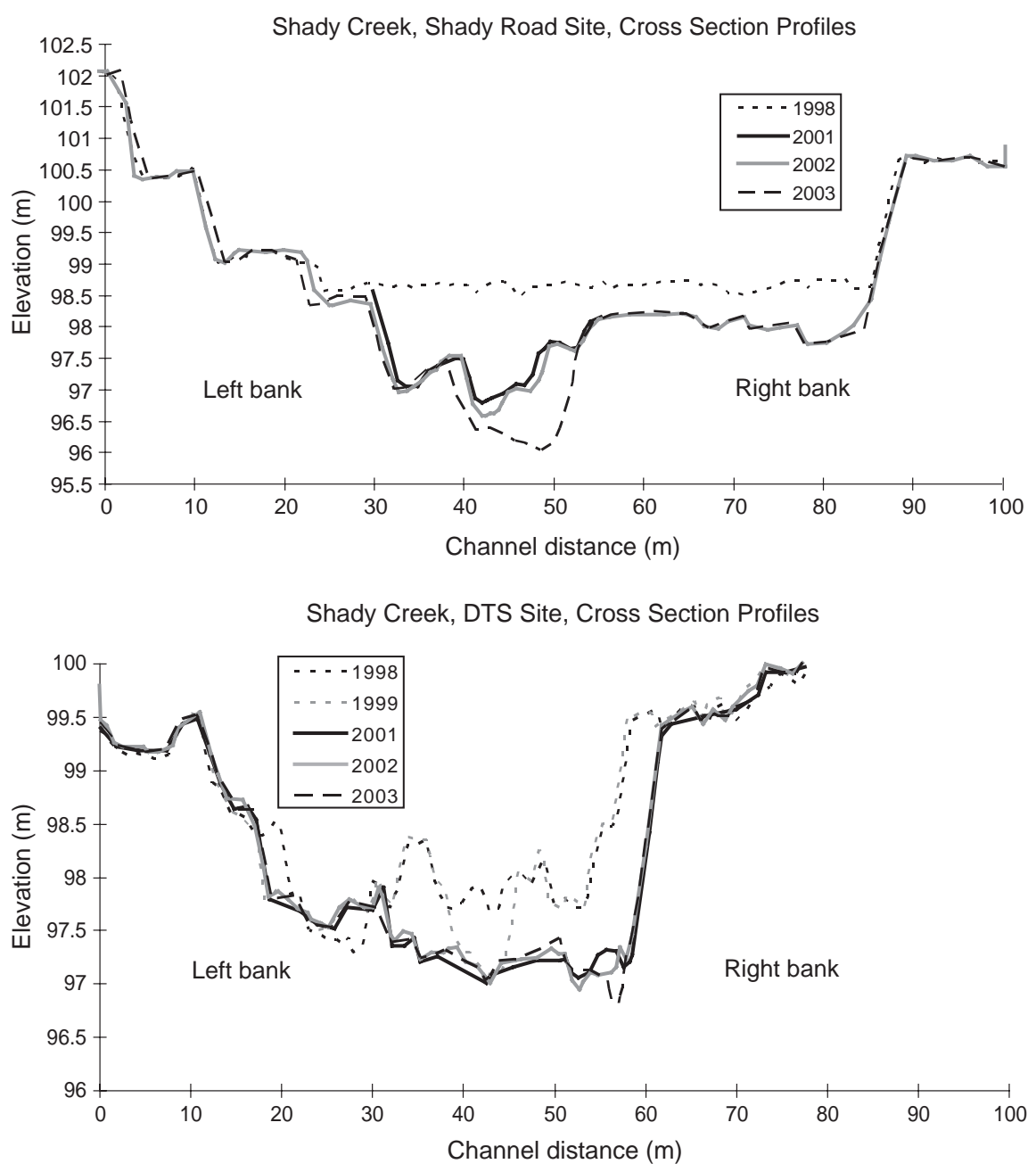

Shady Creek, Rust Pit Site, Cross Section Profiles

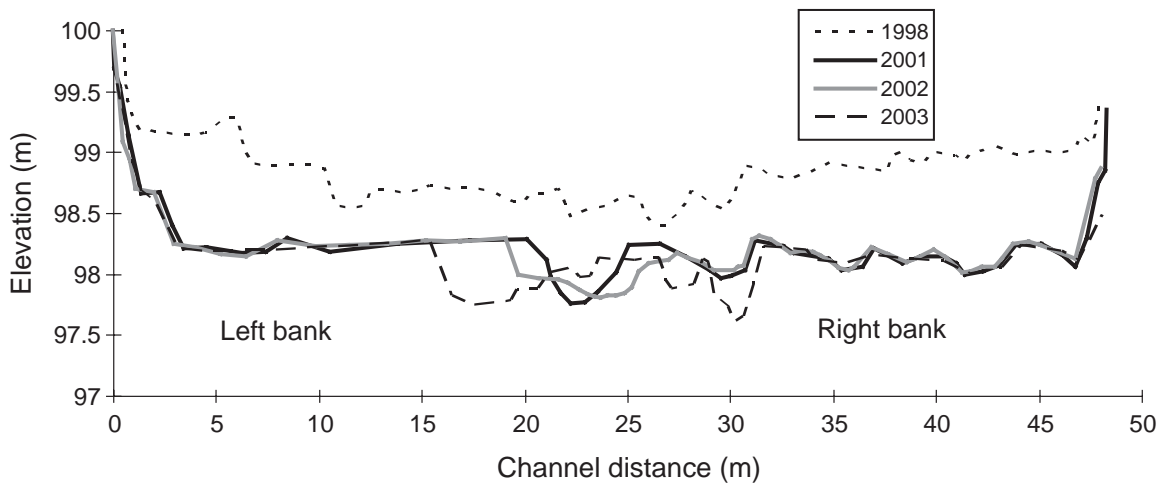

Fig. 6. Repeat cross-sections measured in Shady Creek, a tributary to the SYR impacted by hydraulic mining, from 1998 to 2003. 
derived from hydraulic mining. Although the exact volumes of hydraulic mining sediment were not estimated in this study, we collected channel storage data and repeat cross-sections in impacted reaches to illuminate the significance of stored hydraulic mining sediment. In addition, watershed reconnaissance and review of low elevation digital aerial videography enabled an assessment of the spatial distribution of mainstem sediment storage.

Approximately 500 million $\mathrm{m}^{3}$ of sediment is stored above the thalweg in zero through fifth-order channel networks (Table 4). Debris jams store the majority of sediment in zero-order channels whereas $62 \%$ to $93 \%$ of alluvium in first- through fifth-order channels is stored in well-vegetated terraces that are for the most part inactive and stable but may be susceptible to cutbank erosion. Approximately 12 million $\mathrm{m}^{3}$ of sediment is stored in less stable locations (i.e. debris jams, channel bars, and floodplains) where residence times are much shorter.

Large volumes of hydraulic mining sediment are stored in several upland tributaries, most notably Shady Creek, Spring Creek, Scotchman Creek, and Oregon Creek (Fig. 4) and an extensive glacial outwash terrace is preserved on the mainstem SYR. Additional channel storage data were collected in Shady Creek and on the mainstem SYR to highlight the significance of stored sediment in these reaches (Table 4); however these data were not used in the average storage volume calculations. Storage volumes measured in Shady Creek ( 378 to $676 \mathrm{~m}^{3} / \mathrm{m}$ ) were an order of magnitude greater than the averaged watershed values $\left(6.3\right.$ to $\left.70 \mathrm{~m}^{3} / \mathrm{m}\right)$; and the largest storage volume $\left(2,920 \mathrm{~m}^{3} / \mathrm{m}\right)$ was measured in the SYR glacial outwash terrace reach.

Repeat cross-sections in four upland tributaries (Shady Creek, Rush Creek, Humbug Creek, and Oregon Creek) provided a data set to assess annual channel changes in reaches impacted by hydraulic mining and to document channel erosion processes. Overall from 1998 to 2003 minor erosion and deposition occurred in Rush Creek, Humbug Creek, and Oregon Creek, whereas Shady Creek experienced significant erosion of stored hydraulic mining sediment. Both channel incision and cutbank erosion occurred at the Shady Creek sites. Channel incision dominated cutbank erosion at the Shady Road site and the DTS and Rust Pit sites experienced substantial channel incision and cutbank erosion (Fig. 6). Rates of annual channel change in the four upland tributaries varied from +1.6 to $-17.6 \mathrm{~m}^{2} /$ year with greater erosion occurring during years with above average precipitation (Table 5). Mean annual precipitation at Englebright Lake (Fig. 1) is $85 \mathrm{~cm}$ (http://cdec.water. ca.gov). During the survey period annual precipitation was above average in $1998(145 \mathrm{~cm}), 2000(102 \mathrm{~cm})$, and $2003(93 \mathrm{~cm})$ and below average during $1999(81$ $\mathrm{cm}), 2001(60 \mathrm{~cm})$, and $2002(81 \mathrm{~cm})$.

The relative importance of upland tributary and mainstem sediment sources were further evaluated using low elevation aerial videography of the mainstem channels. The MYR and SYR mainstem channels alternate between bedrock and alluvial reaches. Alluvial reaches store considerable volumes of sediment in the active channel bed, floodplain, and well-vegetated terraces; conversely there is minimal channel storage in bedrock reaches, although patchy alluvium may be stored in deep pools or behind bedrock constrictions and large boulders. The upper segments of the MYR and SYR are primarily bedrock canyons with negligible channel storage. Central portions of the mainstem channels are, for the most part, alluvial with intermittent bedrock reaches; and the lowermost segments are primarily bedrock channels with patchy alluvium stored in localized terraces, floodplains, and channel bars. Central portions of mainstem channels store large quantities of active alluvium, including an extensive glacial outwash terrace on the SYR, and are therefore considered an important sediment source area.

\section{Discussion}

Upper Yuba River hillslope erosion rates are relatively low when compared to the well-documented rates of the Pacific Northwest. Annual sediment production from mass wasting in the Pacific Northwest, one of the most rapidly eroding regions in the United States (Judson and Ritter, 1964), ranges from 9-72 $\mathrm{m}^{3} / \mathrm{km}^{2} /$ year for forested watersheds and $22-$ $3500 \mathrm{~m}^{3} / \mathrm{km}^{2} /$ year for logged and roaded watersheds (Roberts and Church, 1986). In this study, we estimated an annual mass wasting rate of $13 \mathrm{~m}^{3} /$ $\mathrm{km}^{2} /$ year using two sets of aerial photographs spanning 62 years. All 59 active mass wasting sites 
experienced pre-1939 erosion; thus, errors associated with discerning post-1939 erosion are likely high and the mass wasting rates could be significantly inflated. Conversely, the mass wasting inventory is not exhaustive as photo scale, forest canopy, shadows, and photographic parallax prevented identification of small-scale mass movement. Therefore, we infer that the mass wasting rate likely represents a minimum. In addition, this rate does not reflect actual delivery, as one cannot ascertain whether mass movement occurred progressively over a period of years (chronic erosion) or during one or several events (episodic erosion). Even if we assume a 50\% margin of error the annual mass wasting rate would still be considerably lower than rates in more rapidly eroding landscapes such as the Pacific Northwest.

Notable hillslope sediment sources in the upper Yuba River watershed include andesitic mudflows, serpentinized ultramafics, and unvegetated hydraulic mine pits located in Eocene auriferous gravels. The majority $(78 \%)$ of mass wasting in the upper Yuba River occurs in terrain underlain by Tertiary andesitic mudflows (Tvp) or serpentinized ultramafics (um). Large, pre-1939 landslide scarps primarily originate in the andesitic mudflows and generated $70 \%$ of the total sediment attributable to mass wasting. Chronic failures of pre-1939 landslide scarps are associated with ash layers within the mudflow units. These ash layers were altered to low permeability clay layers during post-depositional weathering. Saturation of these clay layers during wet years can trigger widespread mass wasting. In addition, serpentinite units, associated with the Melones Fault Zone (Saucedo and Wagner, 1992), are highly sheared, unvegetated, and extensively eroded by debris slides.

Unvegetated hydraulic mine pits experience continual hillslope erosion, which is readily apparent at mine pits throughout the study area. When largescale hydraulic mining ended in 1884, excavated mine pits had nearly vertical walls (Fig. 2). Repeat photography in 1909, 1953, and 1978 at Malakoff Diggings, located on a tributary to the SYR (Humbug Creek), enabled Yuan (1979) to estimate average annual erosion rates that ranged from 40 to $170 \mathrm{~mm} /$ year for a 69-year period. Assuming an average cliff height of $60 \mathrm{~m}$ and $4 \mathrm{~km}$ of cliff face this equates to an average annual hillslope erosion rate of $\sim 10-40$ million $\mathrm{m}^{3} /$ year for the mine pit at
Malakoff Diggings. Although a significant proportion of the coarser material is deposited in basal fans and on the mine floor, finer sized material moves downstream.

A significant proportion of in-channel storage can be attributed to hydraulic mining. Although exact volumes of mining sediment were not estimated in this study, we collected channel storage volumes and repeat cross-sections within Shady Creek, a tributary to the SYR, to illuminate the significance of stored mining sediment in upland tributaries. Storage volumes in second-, third-, and fourth-order channels within the Shady Creek watershed range from (378$676 \mathrm{~m}^{3} / \mathrm{m}$ ) and are an order of magnitude greater than averaged volumes for the upper Yuba River watershed (6.3 to $70 \mathrm{~m}^{3} / \mathrm{m}$ ). In addition, repeat cross-sections in Shady Creek from 1998 to 2003 documented extensive erosion of stored mining sediment (Fig. 6) and only minor erosion and deposition within less impacted reaches in Rush Creek, Humbug Creek, and Oregon Creek.

Gilbert (1917) predicted the complete removal of mining sediment not in "permanent storage" from tributary channels of the Sierra Nevada foothills by 1960. More recent studies in adjacent watersheds (James, 1989, 1993) recognized that reworking of stored mining sediment will occur asymptotically; and Curtis (1999) further concluded that reworking of stored mining sediment in upland tributaries will likely occur throughout the next millennium. Consequently, we infer mining sediment represents a significant sediment source that will continue to affect long-term sediment yield from the upper Yuba River watershed.

The California Debris Commission (CDC) completed Englebright Dam in December 1940. Its primary purpose was to contain sediment from anticipated future hydraulic mining. Hydraulic mining resumed for a brief period after completion of the dam (James, submitted for publication); however by May 2001 Englebright Lake had accumulated 21.9 million $\mathrm{m}^{3}$ of sediment (Childs et al., 2003; Snyder et al., 2004). One of the primary goals of this paper is to identify major sediment sources in the upper Yuba River and the reservoir sediment volume is an ideal gauge that suggests the relative magnitude of sediment deliveries integrated over the past 62 years.

Average annual Englebright Lake sedimentation from 1940 to 2001 was approximately 0.35 million 
$\mathrm{m}^{3} /$ year; and less than $5 \%$ of lake sedimentation can be attributed to mass wasting. Therefore, the discrepancy between average annual mass wasting and average annual reservoir sedimentation must be filled by twentieth century mining, surface erosion of hillslope sediments, or remobilization of in-channel sediment sources. A period of licensed hydraulic mining from 1893 to 1950 produced approximately 4.8 million $\mathrm{m}^{3}$ of sediment with 1.2 million $\mathrm{m}^{3}$ produced after the closure of Englebright Lake (James, submitted for publication). If we assume $25 \%$ of the pre-1940 licensed mining sediment was conveyed into inchannel storage and transported downstream at a later date then approximately 2 million $\mathrm{m}^{3}$ can be attributed to licensed mining, thus accounting for less than $10 \%$ of Englebright Lake sedimentation.

Field measurements indicate mass wasting dominates surface erosion throughout the study area. Consequently, we infer the gap between mass wasting and Englebright Lake sedimentation is filled primarily by in-channel sediment sources and secondarily by surface erosion and licensed mining. Moreover, we propose that the large volume of sediment stored in active to semi-active channel locations represents the dominant sediment source in the upper Yuba River watershed.

\section{Summary}

This study examines the development of a conceptual model of sediment processes in the upper Yuba River watershed. Field measurements were used to test and revise the conceptual model and were further employed in developing watershed-scale GIS models of hillslope erosion potential and channel storage. The uncalibrated hillslope erosion potential model (Fig. 5) exemplifies the conceptual model in that landscapes with low PET, sparse vegetation, steep slopes, erodible geology and soils, and high road densities are at the greatest risk for hillslope erosion. The channel storage model was calibrated using estimates of channel storage volumes based on stream order and illustrates the conceptual model such that channel storage increases with increasing stream order.

This study differs from the traditional sediment budget approach in that field measurements were not used to define quantitative estimates of erosion, storage, and sediment yield. Rather key sediment source areas and transport processes were identified and used to verify and refine a watershed-scale conceptual model of sediment processes and hypotheses of how to spatially distribute the components of the conceptual model throughout the upper Yuba River watershed were developed using GIS.

The conceptual model of sediment processes and GIS models of hillslope erosion potential and inchannel storage exemplify field measurements:

(i) hillslope erosion rates are relatively low when compared to more rapidly eroding landscapes such as the Pacific Northwest;

(ii) $\sim 5 \%$ of upper Yuba River hillslopes are highly erodible, $10 \%$ are moderately erodible, $28 \%$ are slightly erodible, and $57 \%$ are susceptible to negligible erosion;

(iii) important hillslope sediment sources include andesitic mudflows, serpentinized ultramafics, and hydraulic mine pits;

(iv) hillslope erosion is dominated by mass wasting with surface erosion playing a secondary role;

(v) in-channel sediment storage volumes increase with increasing stream order;

(vi) erosion of in-channel sediment from upland tributaries impacted by hydraulic mining will contribute to long-term upper Yuba River sediment yields;

(vii) the central portion of the study area displays the greatest hillslope erosion potential, stores large quantities of active alluvium including an extensive glacial outwash terrace, and is considered an important sediment source area; and

(viii) in-channel sediment stored in active to semiactive channel locations represents the principal sediment source in the upper Yuba River watershed.

The conceptual and GIS models presented here provide an inventory of sediment dynamics in a dominantly bedrock basin impacted by hydraulic mining and are applicable to northwestern Sierra Nevada rivers that exhibit similar geology, vegetation, soils, and land-use histories. Although field data were extrapolated from small study sites to large areas, the methods used here illustrate the relative importance of 
various sediment processes. Thus, big numbers can be distinguished from little numbers in the conceptual model even if the exact volumes of erosion and transport cannot be measured. Although the assignment of scaling factors and relative multipliers is somewhat subjective, these values represent the field observations and exemplify the conceptual model. Moreover, the GIS models provide first-order approximations of the spatial distribution of hillslope erosion potential and channel storage. Provenance studies using geochemical and magnetic analyses of sediment cores collected in Englebright Lake are in process; and these data may aid in further delineating the relative importance of sediment sources and transport processes and may ultimately enable quantification of the proposed conceptual model.

A conceptual model is a useful tool that facilitates any decision-making system, such as CBDA, that seeks to understand how a landscape functions in a spatially distributed context. Regulators and managers searching for means to limit sediment production or to prioritize habitat restoration projects can benefit from using the approach outlined here. A conceptual model can resolve watershed-scale complexities and can be used as a framework for conducting digital terrain analysis thus enabling spatial approximations. Spatially distributing the components of a conceptual model in a GIS framework provides a guide for developing more detailed sediment budgets or numerical models making it an inexpensive way to develop a roadmap for understanding the linkages among sediment sources and transport processes at a watershed scale.

\section{Acknowledgements}

The CBDA Ecosystem Restoration Program supported this study. We thank Benjamin Wilkins, Elizabeth Gilliam, and Scott Williams for their help in collecting field data. Reviews by Jim O'Connor, Gordon Grant, Allan James, and Ellen Wohl greatly improved the manuscript.

\section{References}

Averill, C.V., 1976. Placer mining for gold in California. California Division of Mines and Geology, Bulletin 135, 93-270.
Barclay, M., 2002. Aerial videography of North, Middle, and South Yuba rivers taped October 22 to 24, 2002: California Dept of Water Resources, three DVD set.

Bateman, P., Wahrhaftig, C., 1966. Geology of the Sierra Nevada. In: Bailey, E.H. (Ed.), Geology of Northern California, California Division of Mines and Geology, Bulletin 190, pp. $105-214$.

Bowie Jr., A.J., 1905. Practical Treatise on Hydraulic Mining. D. Van Nostrand, New York, NY. 13 pp.

Brittan, L.A., 1975. Soil survey of Nevada County area, California. U.S. Department of Agriculture, Soil Conservation Service, Washington, DC.

California Department of Water Resources (CDWR), 1966. Surface Water Hydrology of Yuba-Bear Rivers Hydrographic Unit. Office Report, Sacramento. 96 pp.

California Department of Water Resources (CDWR), 1987. Erosion Control at Malakoff Diggings State Historic Park. Report to the Department of Parks and Recreation Interagency Agreement 0507-075 (DWR 163543), Sacramento. 74 pp.

California Division of Mines and Geology (CDMG), 1999. Factors affecting landslides in forested terrain. California Department of Conservation, Division of Mines and Geology, Sacramento. 5 pp. http://www.consrv.ca.gov/cgs/information/publications/ cgs_notes/note_50/note50.pdf.

Childs, J.R., Snyder, N.P., Hampton, M.A., 2003. Bathymetric and geophysical surveys of Englebright Lake, Yuba-Nevada Counties, California. U.S. Geological Survey Open-File Report OF 03-383 (20 pp., http://www.geopubs.wr.usgs.gov/open-file/ of03-383/).

Christensen, M.N., 1966. Late Cenozoic crustal movements in the Sierra Nevada of California. Geological Society of America Bulletin 77, 162-181.

Clark, W.B., Fuller Jr., W.P., 1968. The original sixteen to one mine. California Division of Mines and Geology (Mineral Information Service) 21 (5), $71-75$.

Costick, L.A., 1996. Indexing current watershed conditions using remote sensing and GIS. Sierra Nevada Ecosystem Project, Center for Water and Wildland Resources, University of California-Davis, Final Report to Congress, 3(3) Davis, CA. 77 pp. http://www.ceres.ca.gov/snep/pubs/v3.html.

Curtis, J.A., 1999. A sediment budget of hydraulic gold-mining sediment, Steephollow Creek basin, California, 1853-1997. MS Thesis, Geology Dept., Humboldt State University, Arcata, CA. $94 \mathrm{pp}$.

Davis, F.W., Stoms, D.M., 1996. Sierran vegetation: A gap analysis. Sierra Nevada Ecosystem Project. Center for Water and Wildland Resources, University of California, Davis, CA, Final Report to Congress, 2, 671-689. http://gapanalysis.nbii.gov/.

Dietrich, W.E., Dunne, T., 1978. Sediment budget for a small catchment in mountainous terrain. Zeitschrift für Geomorphologie 29, 191-206.

Ferguson, H.G., Gannett, R.W., 1932. Gold quartz veins of the Alleghany District California. U.S. Geological Survey Professional Paper 172 (139 pp.).

Flint, A.L., Childs, S.W., 1987. Calculation of solar radiation in mountainous terrain. Journal of Agricultural and Forest Meteorology 40, 233-249. 
Flint, L.E., Guay, J.R., Flint, A.L., Curtis, J.A., Alpers, C.N., 2004. Spatially distributed model of flow and sediment transport in the upper Yuba River watershed. 3rd Biennial CALFED Bay-Delta Science Conference Abstract, October 4-6, 2004, Sacramento, CA

Gilbert, G.K., 1917. Hydraulic-mining debris in the Sierra Nevada. U.S. Geological Survey Professional Paper 105 (154 pp.).

Hall, W.H., 1880. Mining debris in the Sacramento River. Report of the State Engineer to Legislature, House Ex Doc 69, 46th Congress, 2nd session. 128 pp.

Hanes, R.O., 1994. Soil survey of Tahoe National Forest area, California. U.S. Department of Agriculture, Forest Service, Washington, DC.

James, L.A., 1989. Sustained storage and transport of hydraulic mining sediment in the Bear River, California. Annals AAG 79 (4), 570-592.

James, L.A., 1993. Sustained reworking of hydraulic mining sediment in California: G.K. Gilbert's sediment wave model reconsidered. Zeitschrift für Geomorphologie 88, 49-66.

James, L.A., 2005. Sediment from hydraulic mining detained by Englebright and small dams in the Yuba Basin. Geomorphology (submitted for publication)

James, L.A., Harbor, J., Fabel, D., Dahms, J., Elmore, D., 2002. Late Pleistocene glaciations in the Northwestern Sierra Nevada, California. Quaternary Research 57 (3), 409-419.

Judson, S., Ritter, D.F., 1964. Rates of regional denudation in the United States. Journal of Geophysical Research 69 (16), 3395-3401.

Kasai, M., Marutani, T., Brierley, G.J., 2004. Patterns of sediment slug translation and dispersion following typhoon-induced disturbance, Oyabe Creek, Kyushu, Japan. Earth Surface Processes and Landforms 29, 59-79.

Kattelman, R., 1996. Hydrology and Water Resources. Sierra Nevada Ecosystem Project, Center for Water and Wildland Resources, University of California-Davis, Final Report to Congress, 2(30) Davis, CA, 66 pp. http://www.ceres.ca.gov/ snep/pubs/v2s3.html.

Kelsey, H.M., 1980. A sediment budget and an analysis of geomorphic process in the Van Duzen River basin, north coastal California, 1941-1975. Geological Society of America Bulletin 91, 1119-1216.

Kelsey, H.M., Lamberson, R., Madej, M.A., 1987. Stochastic model for the long-term transport of stored sediment in a river channel. Water Resources Research 23 (9), 1738-1750.

Lindgren, W., 1911. Tertiary gravels of the Sierra Nevada of California. U.S. Geological Survey Professional Paper 73 (226 pp.).

Macklin, M.G., Lewin, J., 1989. Sediment transfer and transformation of an alluvial valley floor: the River South Tyne, Northumbria, UK. Earth Surface Processes and Landforms 14, $223-246$.

Madej, M.A., Ozaki, V., 1996. Channel response to sediment wave propagation and movement, Redwood Creek, California, USA. Earth Surface Processes and Landforms 21, 911-927.

May, P.R., 1970. Origins of hydraulic mining. Holmes Book Co., Oakland, CA. 88 pp.
Nelson, E.J., Booth, D.B., 2002. Sediment sources in an urbanizing, mixed land-use watershed. Journal of Hydrology 264, 51-68.

Reid, L.M., Dunne, T., 1996. Rapid Evaluation of Sediment Budgets. Catena Verlag, Reiskirchen, Germany. 164 pp.

Roberts, R.G., Church, M., 1986. The sediment budget in severely disturbed watersheds, Queen Charlotte Ranges, British Columbia. Canadian Journal of Forest Research 16, 1092-1106.

Saucedo, G.J., Wagner, D.L., 1992. Geologic Map of the Chico Quadrangle, Scale 1:250,000. California Dept. of Mines and Geology RGM007A.

Selby, M.J., 1993. Hillslope Materials and Processes. Oxford University Press, New York, NY. 449 pp.

Shroder, J.F., Bishop, M.P., 2003. A perspective on computer modelling and fieldwork. Geomorphology 53, 1-9.

Singh, V.P., 1995. Computer Models of Watershed Hydrology. Water Resources Publications, Highlands Ranch, CO. 1130 pp.

Snyder, N.P., Rubin, D.M., Alpers, C.N., Childs, J.R., Curtis, J.A., Flint, L.E., Wright, S.A., 2004. Estimating accumulation rates and physical properties of sediment behind a dam: Englebright Lake, Yuba River, northern California. Water Resources Research 40, W11301.

Trimble, S.W., 1983. A sediment budget for Coon Creek basin in the Driftless Area, Wisconsin, 1853-1997. American Journal of Science 283, 454-474.

Turner, F.C., 1891. Reports of Mr. F. C.Turner, Asst. Engineer, of reconnaissance of Feather, Yuba, Bear, and American rivers above the Foot Hills, and of deposits of mining gravel subject to hydraulic process in their basins. Appendix VV, House Doc 1, Part 2, 52nd Congress, 1st session: 3041-3087.

U.S. Department of Agriculture - Natural Resource Conservation Service (USDA-NRCS), 1994. State Soil Geographic (STATSGO) Data Base —Data use information: Misc. Pub. 1492. http://www.ftw.nrcs.usda.gov/stat_data.html.

Wakabayashi, J., Sawyer, T.L., 2001. Stream incision, tectonics, uplift, and evolution of topography of the Sierra Nevada, California. Journal of Geology 109 (5), 539-562.

Walling, D.E., 1983. The sediment delivery problem. Journal of Hydrology 65, 209-237.

Western Regional Climate Center (WRCC), 2003. Western U.S. Historical Summaries by State, Annual Precipitation Averages and Extremes. http://www.wrcc.dri.edu/htmlfiles/ca/ca.ppt.ext. html.

Whitney, J.D., 1880. The auriferous gravels of the Sierra Nevada. Memoir of the Museum of Comparative Zoology 6 (569 pp.).

Wildman, N., 1981. Episodic removal of hydraulic mining-debris, Yuba and Bear River basins, California. MS Thesis, Dept. of Earth Science, Colorado State University, Fort Collins, CO. $107 \mathrm{pp}$.

Yeend, W.E., 1974. Gold-bearing gravel of the ancestral Yuba River, Sierra Nevada, California. U.S. Geological Survey Professional Paper 772 (44 pp.).

Yuan, G., 1979. The geomorphic development of an hydraulic mining site in Nevada County, California. MS Thesis, Applied Earth Sciences Dept. Stanford University, Stanford, California. 56 pp. 